Research Paper

\title{
Long non-coding RNAs could act as vectors for paternal heredity of high fat diet-induced obesity
}

This article has been corrected. Correction in: Oncotarget. 2019; 10:798-798.

Tian An ${ }^{1, *}$, Teng Zhang ${ }^{2, *}$, Fei Teng ${ }^{1}$, Jia-Cheng Zuo ${ }^{1}$, Yan-Yun Pan ${ }^{1}$, Yu-Fei Liu ${ }^{3}$, Jia-Nan Miao ${ }^{1}$, Yu-Jie Gu${ }^{1}$, Na Yu ${ }^{1}$, Dan-Dan Zhao ${ }^{1}$, Fang-Fang Mo ${ }^{1}$, Si-Hua Gao ${ }^{1}$ and Guangjian Jiang ${ }^{1}$

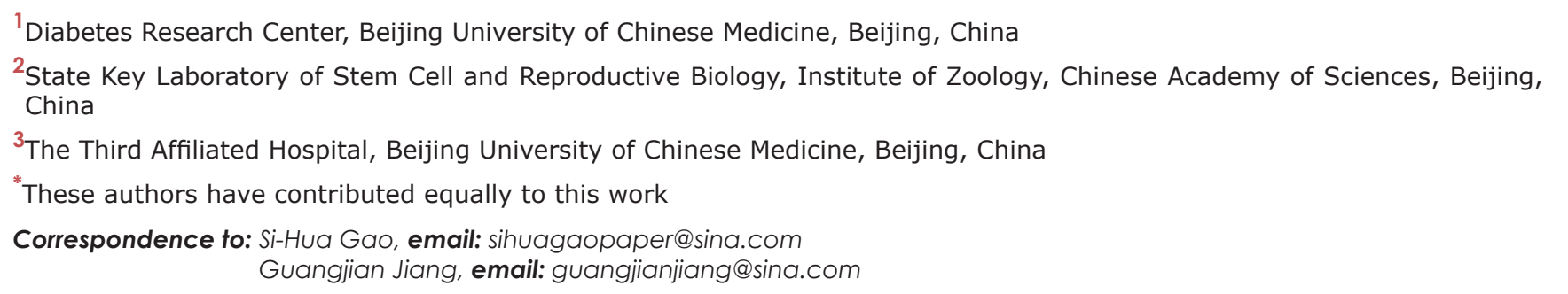
Keywords: long non-coding RNA, paternal inheritance, genetic vector, obesity

Received: April 03, $2017 \quad$ Accepted: April 24, $2017 \quad$ Published: May 24, 2017

Copyright: An et al. This is an open-access article distributed under the terms of the Creative Commons Attribution License 3.0 (CC BY 3.0), which permits unrestricted use, distribution, and reproduction in any medium, provided the original author and source are credited.

\section{ABSTRACT}

Long non-coding RNAs (IncRNAs) play an important role in epigenetic regulation, and abnormalities may lead to male infertility. To investigate whether IncRNAs are involved in intergenerational inheritance of obesity and obesity-induced decline in fertility, we divided mice into obesity (FO mice fed a high-fat diet, F0-HFD) and nonobese (FO mice fed normal chow, FO-NC) model groups and their male offspring (F1HFD and F1-NC, respectively). We examined the differences in the expression levels of IncRNAs and mRNAs in the FO-HFD/F0-NC and F1-HFD/F1-NC groups. The results revealed similar expression patterns in the F1-HFD/F0-HFD groups at both the IncRNA and mRNA levels. The maximum difference in the IncRNA expression was observed between the FO-HFD and FO-NC groups. The differentially expressed IncRNA targets and mRNAs identified in our study are mainly involved in GnRH signalling pathway, metabolic process, and Hippo signalling pathway; similarly expressed IncRNAs and mRNAs in F1-HFD/F0-HFD are closely linked with G-protein coupled receptor signalling pathway, pancreatic polypeptide receptor activity, and lysine biosynthesis, which may play an important role in the molecular mechanism of intergenerational inheritance of obesity. Furthermore, potential genes that might play important roles in the pathogenesis of obesity-related low fertility were revealed by IncRNA-and mRNA-interaction studies based on the microarray expression profiles. In conclusion, we found that IncRNA could be involved in obesity-induced infertility by expressing abnormalities, which could act as genetic vectors of paternal inheritance of obesity.

\section{INTRODUCTION}

Over the past 30 years, obesity in males in the reproductive age has tripled, concomitant with the increase in male infertility [1]. Obesity can have a negative impact on male reproductive potential by reducing sperm density and motility [2, 3] and increasing the release of reactive oxygen species (ROS), which can cause DNA damage and influence plasma membrane integrity in sperm [4, 5]. Male offspring from high fat diet (HFD) fathers have a high sensitivity to HFD-induced metabolic and reproductive disturbances [6]. Genetic and epigenetic changes in the spermatozoa may explain paternal programming 
of offspring phenotypes induced by paternal obesity [7]. Inter/transgenerational phenotype transmission is associated with histone modifications, altered DNA methylation, and noncoding RNA transcripts [8-10]. DNA methylation is required for spermatogenesis, and it has been demonstrated that diet-induced paternal obesity regulates germ cell methylation status and causes metabolic disorders in two generations of mice $[11,12]$. Paternal obesity changes the expression of insulin-like growth factor 2 (IGF2) in infants by influencing normal IGF2 methylation in spermatozoa [13]. The contribution of the patrilineal phenotype to fertility is not just at the DNA level [14]; sperm RNA can also reflect the quality of spermatogenesis, and its fertilizing capacity and post-fertilisation functions [15].

Long non-coding RNAs (lncRNAs) play an important role in mature sperm. LncRNAs can play a critical role in genomic imprinting [16], adipogenesis and metabolism [17], adipocyte differentiation and development [18]. Interestingly, lncRNA may also play an important role in the context of the expanding sperm RNA-protein network [19, 20]. Our previous study revealed that $7721 \mathrm{lncRNAs}$ are differentially expressed between normal and diabetic sperm groups, suggesting that lncRNA may be a potential regulatory target associated with spermatogenesis in men with diabetes [21]. Cadmium (Cd) exposure can lead to abnormal changes in the expression profile of sperm lncRNAs [22]. Another study found that obesity might be associated with the expression of disorderslncRNA by constructing a circulating lncRNA expression profile in obese and nonobese humans [23].

Although the role of lncRNA in obesity and reproduction has been investigated previously, few studies have examined the role of lncRNA in the context of obesity-induced infertility, and in particular, its influence on offspring spermatogenesis. Therefore, we investigated lncRNAs that are differentially expressed in the spermatozoa of obese and non-obese mice and their offspring by microarray analysis, in order to study the impact of obesity on mice fertility and intergenerational inheritance from lncRNA expression. This study provides a possible lncRNA-related mechanism for obesity-induced male sterility.

\section{RESULTS}

\section{High fat diet-induced obesity altered the expression of IncRNA and mRNA in mouse sperm}

To identify lncRNAs and mRNAs that are differentially expressed in mice sperm between the obese and normal groups, we carried out microarray analysis of lncRNA and mRNA expression in F0-HFD and F0-
NC. In total, 6,354 lncRNAs and 3,370 mRNAs were upregulated while 7,541 lncRNAs and 5,655 mRNAs were downregulated. These results demonstrated that a high-fat diet induced obvious changes in the lncRNA and mRNA expression profiles in the spermatozoa of obese mice. The scatter plot and top 15 differentially expressed lncRNAs and mRNAs between F0-HFD and F0-NC are shown in Figure $1 \mathrm{~A}$ and Table 1.

\section{The expression patterns of LncRNA and mRNA in male offspring (F1) spermatozoa of obese and normal mice were significantly different}

In order to reveal the impact of obesity induced by a high-fat diet on progeny fertility, we examined the expression of IncRNA and mRNA in the spermatozoa of obese and normal mouse offspring. Microarray analysis showed that 5,984 lncRNAs and 2,661 mRNAs were upregulated and 5,543 $\mathrm{lncRNAs}$ and 5,846 mRNAs were downregulated in F1-HFD relative to F1-NC mice (Figure 1B). The top 15 significantly differentially expressed lncRNAs and mRNAs in F1-HFD/F1-NC are listed in Table 2.

\section{LncRNA may act as the hereditary vector inducing paternal heredity of obesity (HFD)}

To further determine whether intergenerational inheritance of obesity occurs via the expression of sperm lncRNA, we compared lncRNA expression in the spermatozoa and found that 26,531 lncRNAs in F1 and F0 obese mice had the same expression profiles. The opposite of differentially expressed lncRNA is a minority, 1,752 lncRNAs and 1,218 mRNAs were upregulated and 2,160 lncRNAs and 2,382 mRNAs were downregulated in F1-HFD as compared to those in F0HFD mice (Figure 1C).

\section{Functional analysis of differentially expressed genes}

A Gene Ontology (GO) analysis of differentially expressed mRNAs was carried out to determine their functional significance [24] (Supplementary Table 1). Upregulated transcripts in the F0-HFD/F0-NC and F1HFD/F1-NC groups were enriched in "spermatogenesis, exchange of chromosomal proteins (biological process)", "sperm fibrous sheath (cellular component)" while downregulated transcripts were most highly enriched in "MHC class II protein complex" and "positive regulation of deacetylase activity" (Figure 2). The most highly enriched GO terms associated with similarly expressed transcripts in the F1-HFD/F0HFD groups were "positive regulation of somatostatin secretion", "steroid delta-isomerase activity" and "collagen type IV" (Figure 3). 
Table 1: Top 15 differentially expressed IncRNAs and mRNAs in F0-HFD/F0-NC groups

\begin{tabular}{|c|c|c|c|}
\hline \multicolumn{2}{|c|}{ mRNAs } & \multicolumn{2}{|c|}{ IncRNAs } \\
\hline Gene_name & Fold change & Gene_name & Fold change \\
\hline Ngp & 428.3283623 & NONMMUG009536 & 24.29140951 \\
\hline Mcam & 20.25591007 & NONMMUG029430 & 24.17902223 \\
\hline Gja5 & 19.53615843 & NONMMUG033851 & 24.11090336 \\
\hline Rhd & 19.44082314 & NONMMUG018726 & 24.02573231 \\
\hline Fgg & 19.42386373 & ENSMUSG00000084785 & 22.89749706 \\
\hline Yaeld1 & 18.88614046 & Mir32 & 22.30121644 \\
\hline Gm5148 & 18.83236784 & ENSMUSG00000090026 & 22.15050983 \\
\hline Rpl6 & 18.38511123 & NONMMUG011486 & 22.1074696 \\
\hline 9530003J23Rik & 18.33321626 & NONMMUG024207 & 21.4604214 \\
\hline Fgf15 & 17.88020039 & NONMMUG035045 & 21.3527503 \\
\hline Agl & 17.79835497 & NONMMUG037260 & 21.15388882 \\
\hline 3110035E14Rik & 17.22059397 & NONMMUG031523 & 20.44854092 \\
\hline Yipf2 & 17.13240759 & NONMMUG003313 & 20.29794122 \\
\hline Golt1a & 17.11676312 & Mir206 & 20.05179104 \\
\hline $\mathrm{Gc}$ & 17.00442951 & ENSMUSG00000097045 & 19.88438449 \\
\hline B4galt5 & 0.032368167 & NONMMUG016700 & 0.047472897 \\
\hline Zfp52 & 0.031937927 & NONMMUG017643 & 0.047422387 \\
\hline Mmp13 & 0.031746956 & NONMMUG017032 & 0.046587816 \\
\hline $\operatorname{Irg} 1$ & 0.031315495 & AI504432 & 0.045872836 \\
\hline Slc7a11 & 0.030710923 & NONMMUG010104 & 0.045184993 \\
\hline Adm & 0.024873517 & NONMMUG016696 & 0.045036318 \\
\hline Tnfrsf11b & 0.024284736 & AI504432 & 0.044699013 \\
\hline Hcar2 & 0.023393394 & NONMMUG016242 & 0.043215483 \\
\hline Serpinb2 & 0.022907985 & NONMMUG016698 & 0.042536917 \\
\hline Lif & 0.022141803 & ENSMUSG00000097418 & 0.042404113 \\
\hline Hmox1 & 0.022031532 & Mcpt-ps1 & 0.038230371 \\
\hline Illa & 0.021840103 & Gm14023 & 0.037605847 \\
\hline $\mathrm{Il} 10$ & 0.021072211 & NONMMUG005850 & 0.023176845 \\
\hline Slc $7 \mathrm{a} 2$ & 0.007905572 & NONMMUG038489 & 0.022920052 \\
\hline $\mathrm{Cxcl3}$ & 0.001159635 & NONMMUG008794 & 0.011994736 \\
\hline
\end{tabular}

Kyoto Encyclopedia of Genes and Genomes (KEGG) pathway analysis

Ten pathways were significantly enriched among the differentially expressed transcripts $(P<0.05$; Supplementary Table 2). The most highly enriched pathway in the F0-HFD/F0-NC groups was "osteoclast differentiation", and the most enriched pathways in the F1-
HFD/F1-NC groups were "metabolic pathways" (Figure 4). Similarly expressed transcripts in the F1-HFD/F0-HFD groups were highly enriched in "G-protein coupled receptor signalling pathway", "pancreatic polypeptide receptor activity", and "Lysine biosynthesis" (Figure 3). Many of these pathways were linked to obesity, such as "B cell receptor signalling pathway", "MAPK signalling pathway", "GnRH signalling pathway", "Hippo signalling pathway", 

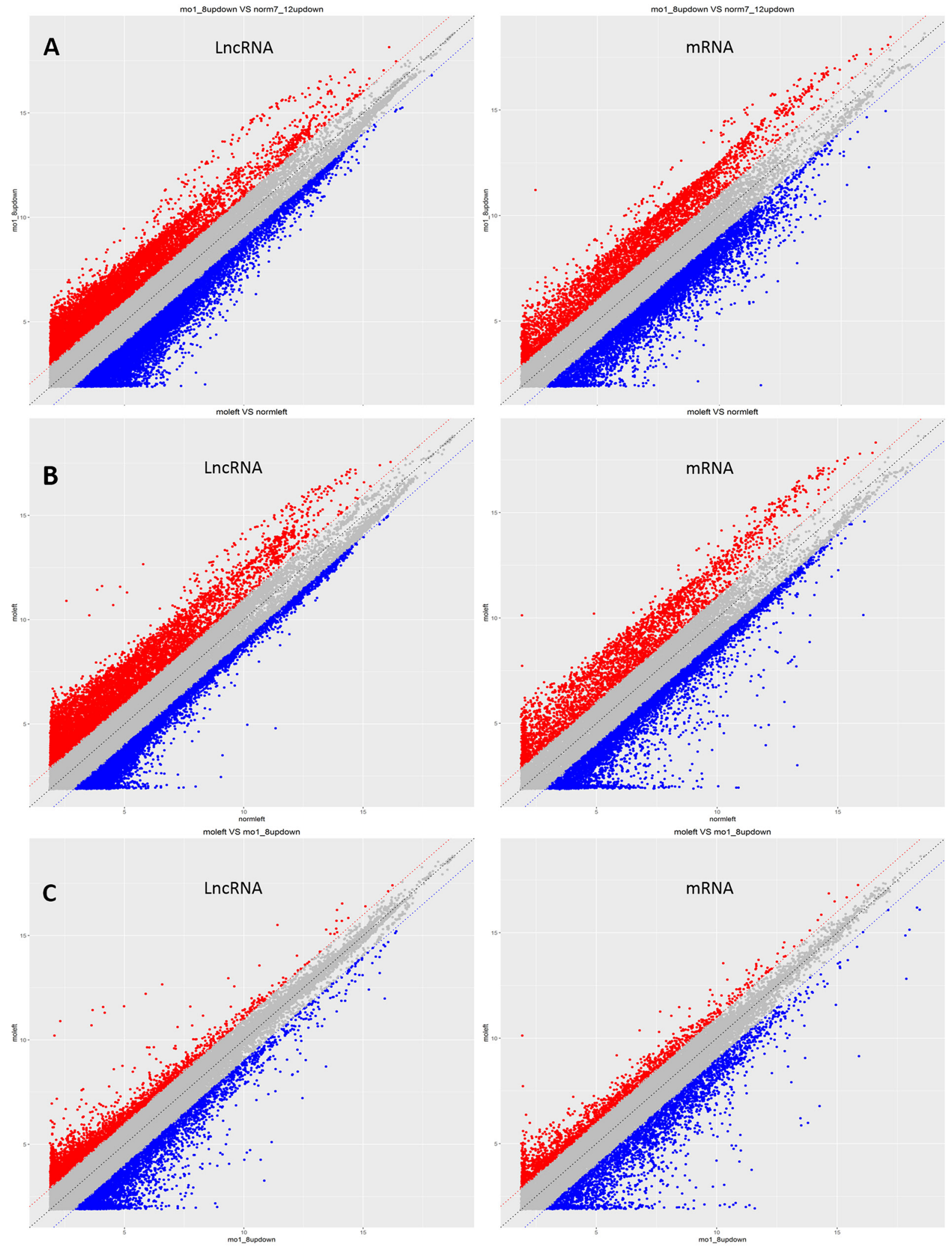

Figure 1: LncRNA scatter plots used to identify differentially expressed IncRNAs in four groups. $X$ and $Y$ axes represent averaged normalised signal values of the control and experimental group microarray samples. LncRNAs above the top red line and below the bottom blue line showed greater than 2.0-fold changes in expression between the two compared groups. (A) F0-HFD/F0-NC; (B) F1HFD/F1-NC; and (C) F1-HFD/F0-HFD. 
Table 2: Top 15 differentially expressed IncRNAs and mRNAs in F1-HFD/F1-NC groups

\begin{tabular}{|c|c|c|c|}
\hline \multicolumn{2}{|c|}{ mRNAs } & \multicolumn{2}{|c|}{ IncRNAs } \\
\hline Gene_name & Fold change & Gene_name & Fold change \\
\hline Scnn $1 b$ & 55.989485 & NONMMUG003816 & 187.63459 \\
\hline ВС048679 & 39.563688 & E030003E18Rik & 117.004727 \\
\hline $\mathrm{C} 8 \mathrm{~b}$ & 18.183759 & NONMMUG012756 & 108.169053 \\
\hline Prss56 & 17.354256 & NONMMUG004111 & 25.9867966 \\
\hline Hpgds & 17.036948 & 1700023F02Rik & 22.7352112 \\
\hline Nutm1 & 16.77879 & 1700047L14Rik & 21.3072219 \\
\hline Fgf15 & 16.701433 & ENSMUSG00000086534 & 20.1978776 \\
\hline Rpl6 & 16.6689 & NONMMUG040279 & 19.4705563 \\
\hline Dnah10 & 16.504961 & NONMMUG008279 & 18.7480326 \\
\hline Ptprcap & 15.671903 & NONMMUG016872 & 18.7032969 \\
\hline Ush1c & 15.39363 & NONMMUG013706 & 18.213956 \\
\hline E2f3 & 14.918401 & NONMMUG011486 & 18.0963333 \\
\hline Agl & 14.652562 & NONMMUG018726 & 18.033556 \\
\hline Ssty1 & 14.198563 & ENSMUSG00000090026 & 17.7970473 \\
\hline Lrrc71 & 14.059018 & ENSMUSG00000099387 & 17.5358967 \\
\hline Ank1 & 0.0093276 & NONMMUG005810 & 0.06008047 \\
\hline Sparcl1 & 0.0091043 & ENSMUSG00000084421 & 0.06005461 \\
\hline Synpo2 & 0.0083851 & NONMMUG023650 & 0.04962984 \\
\hline Pdlim3 & 0.0077229 & NONMMUG044366 & 0.0490406 \\
\hline Sh3bgr & 0.0076126 & NONMMUG023650 & 0.04353695 \\
\hline $\operatorname{Pgm} 5$ & 0.0071856 & ENSMUSG00000085779 & 0.04183152 \\
\hline Smoc2 & 0.0067167 & ENSMUSG00000097250 & 0.03925611 \\
\hline Col12a1 & 0.0067059 & NONMMUG034856 & 0.03851915 \\
\hline $\operatorname{Pgm} 5$ & 0.0059943 & NONMMUG025408 & 0.02739993 \\
\hline Tagln & 0.0038105 & NONMMUG029313 & 0.02460214 \\
\hline Cxcl14 & 0.0037608 & ENSMUSG00000097324 & 0.02170222 \\
\hline Acta1 & 0.0030556 & Mir143hg & 0.02041349 \\
\hline Pcp411 & 0.0021702 & NONMMUG012048 & 0.01576866 \\
\hline Pcp4 & 0.0020343 & NONMMUG034976 & 0.01074036 \\
\hline Myh11 & 0.0007876 & NONMMUG012048 & 0.0103487 \\
\hline
\end{tabular}

and "fatty acid metabolism". The number of genes in the top ten pathways significantly enriched among the differentially expressed transcripts are listed in Figure 4.

\section{Coding-non-coding gene co-expression network}

The majority of the identified transcripts have unknown function. We selected four (NONMMUG009536, Malat1, NONMMUG029444, NONMMUG034976) lncRNAs for cis and trans target gene prediction. A coding-non-coding gene co-expression network was constructed from re-annotated Affymetrix Mouse Genome Array data (Figure 5).

\section{Quantitative real-time (qRT)-PCR validation}

Eight lncRNAs were chose for verification of the microarray results in three groups of samples by quantitative real-time PCR. qRT-PCR assay showed that 
the expression of lncRNA miR-6937, 1700009J07Rik, H19, and ENSMUSG00000090026 were upregulated, whereas nuclear-enriched abundant transcript (Neat)1, metastasisassociated lung adenocarcinoma transcription (Malat)1, and small nucleolar RNA (Snora)47 were downregulated. This result is consistent with the expression profiles from the microarray analysis (Figure 6). Hence, the qRT-PCR data verified the microarray results.

\section{DISCUSSION}

Increasing evidence indicates that obesity induced by a high-fat diet can be inherited from the father, but the nature of the genetic vector inducing this newly acquired phenotype remains unclear. Identification of the genetic vector involved in obesity is an important step in addressing obesity. Our study showed that there was a
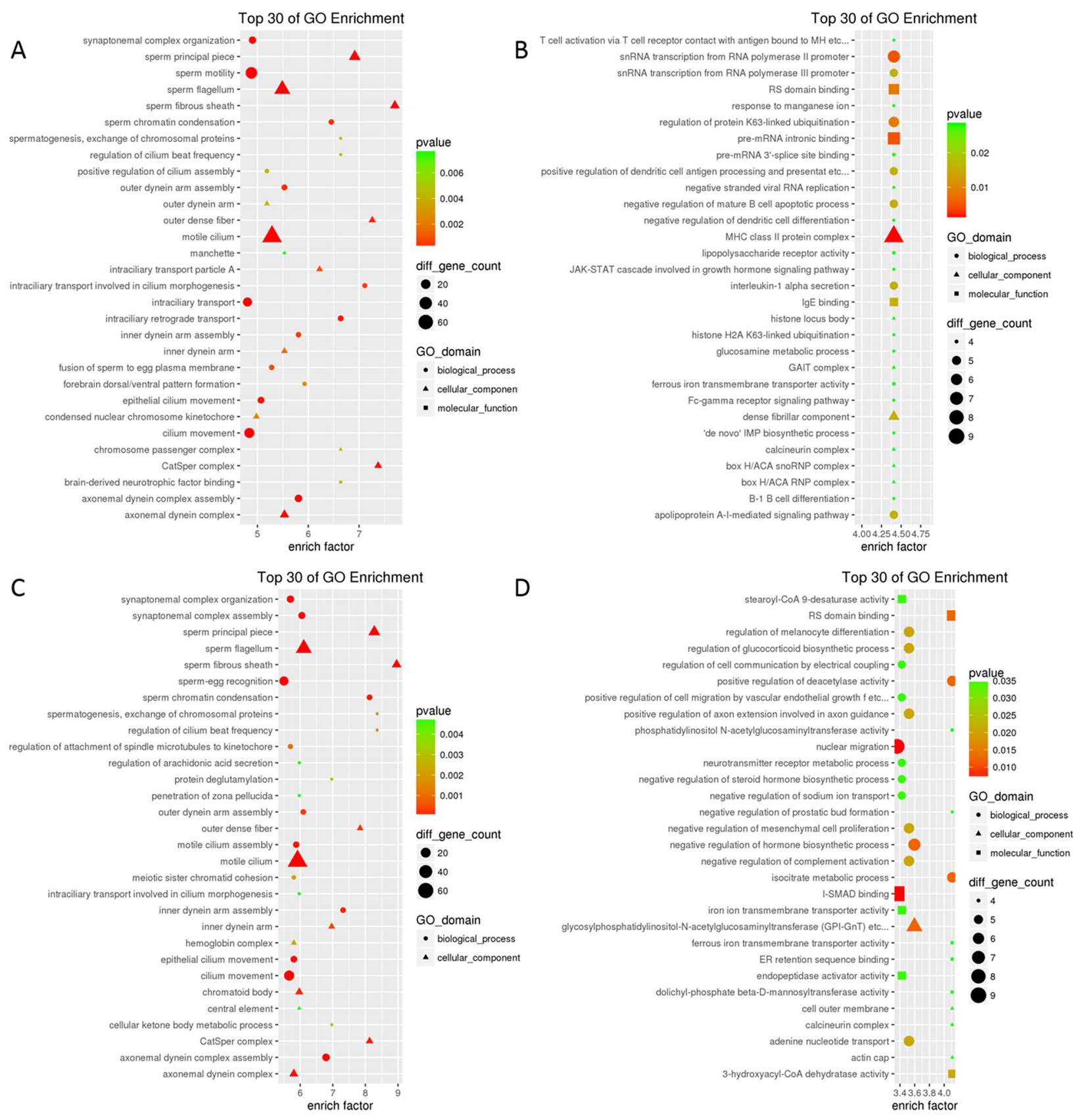

Figure 2: Top 30 enriched GO terms for differentially expressed mRNAs. The terms were divided into three categories, including biological process (BP, circles), cellular component (CC, triangles), and molecular function (MF, squares). Size represents the number of enriched genes; colour indicates the degree of enrichment. F0-HFD/F0-NC group (A) upregulated; (B) downregulated; and F1HFD/F1-NC group (C) upregulated; (D) downregulated. 
significant difference in the expression of lncRNAs and mRNAs in spermatozoa of high-fat diet-induced obese mice and normal mice, and these differences were also present in their male offspring (F1). Interestingly, the opposite expression pattern was found in the F1-HFD and F0-HFD groups, where minimal differential expression of lncRNA and mRNA was observed. In other words, similar expression patterns of IncRNA and mRNA were retained in the F1-HFD/F0-HFD groups, suggesting that lncRNA acts as the hereditary vector inducing paternal inheritance of obesity.

Research has shown that RNA functions as a vector of epigenetic information [25]. However, their inheritance mechanism in the spermatozoa of obese mice remains unclear. LncRNAs are critical for spermatogenesis, and their dysregulation might lead to male infertility [22]. However, only a few lncRNAs have been studied in obese male mice. NEAT1 is expressed in human embryonic stem cells [26] and is involved in the regulation of spermatogenesis [27]. We found in our microarray analysis that the NEAT1 and Malat1 expression levels were downregulated in obese as compared to non-obese mice (NEAT1: F0-HFD/F0-NC $=0.18, \mathrm{~F} 1-\mathrm{HFD} / \mathrm{F} 1-\mathrm{NC}$ $=0.4$; Malat $1:$ F0-HFD/F0-NC $=0.21$, F1-HFD/F1-NC $=0.35)$, which were similar to previously reported values [27]. Decreased expression of NEAT1 is associated with reduced sperm quality and low fertility, and its expression is negatively regulated by Malat1 [27]. Together, these results suggest that high-fat diet-induced dysregulation of lncRNA expression promotes epigenetic changes that can remain phenotypically silent but are later transmitted to the progeny.

The GO analysis showed that lncRNAs that were differentially expressed between the F0-HFD and F0$\mathrm{NC}$ groups were mostly highly enriched in functions associated with obesity-related reproductive pathogenesis, and the same differences were observed in their offspring. Specifically, "osteoclast differentiation" and "B cell receptor signalling pathway" have been linked to obesity, which is detrimental to bone formation and bone mineral density [28, 29]. Investigating the relationship between bone and fat metabolism can not only clarify the mechanisms common to obesity and osteoporosis, but also identify lncRNAs and other molecules associated with osteoclast differentiation that can serve as therapeutic targets in the treatment of these disorders. Furthermore, in the F1-HFD/F0-HFD groups, similarly expressed lncRNAs and mRNAs were mostly highly enriched in "positive regulation of somatostatin secretion", "positive regulation of somatostatin secretion", "steroid delta-isomerase activity", and "integral component of membrane" GO terms. Thus, intergenerational inheritance of obesity may have occurred through mechanisms mediated by the similarly expressed mRNAs and lncRNAs.

It has been demonstrated in other species, that gonadotropin-releasing hormone $(\mathrm{GnRH})$ signalling inputs could promote male mating-like behaviour [30]. The GnRH signalling pathway plays a key role in the central regulation of reproduction [31], and can regulate vital
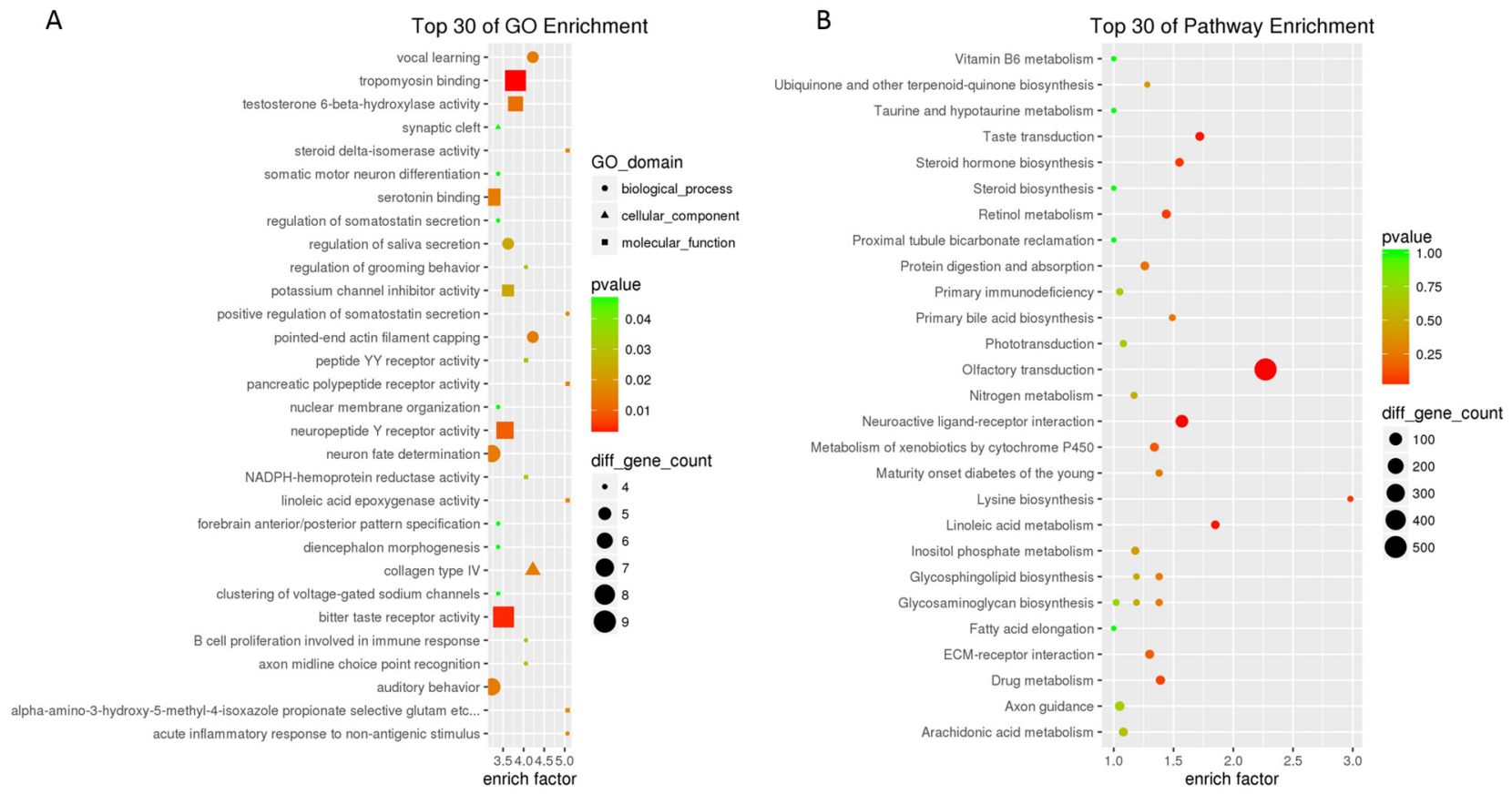

Figure 3: Top 30 enriched GO terms (A) and KEGG pathways (B) for similarly expressed mRNAs in the F1-HFD and F0HFD groups. 
factors associated with fertility, such as leptin, insulin and ghrelin, which have been identified as the key mediators of "vital metabolic control" [32]. The Hippo signalling pathway represents a new molecular target for the regulation of organ-growth functional remodelling [33]. In the present research, we found that in the high-fat/normal groups, whether in the F0 or F1 generation, transcripts associated with GnRH and Hippo signalling pathway were upregulated, suggesting that the biological processes and regulatory mechanisms participating in the $\mathrm{GnRH}$ and Hippo signalling pathways may represent potential targets for the treatment of male infertility in obesity induced by a high-fat diet. Additionally, similarly expressed mRNAs in F1-HFD/F0-HFD were found to be closely linked

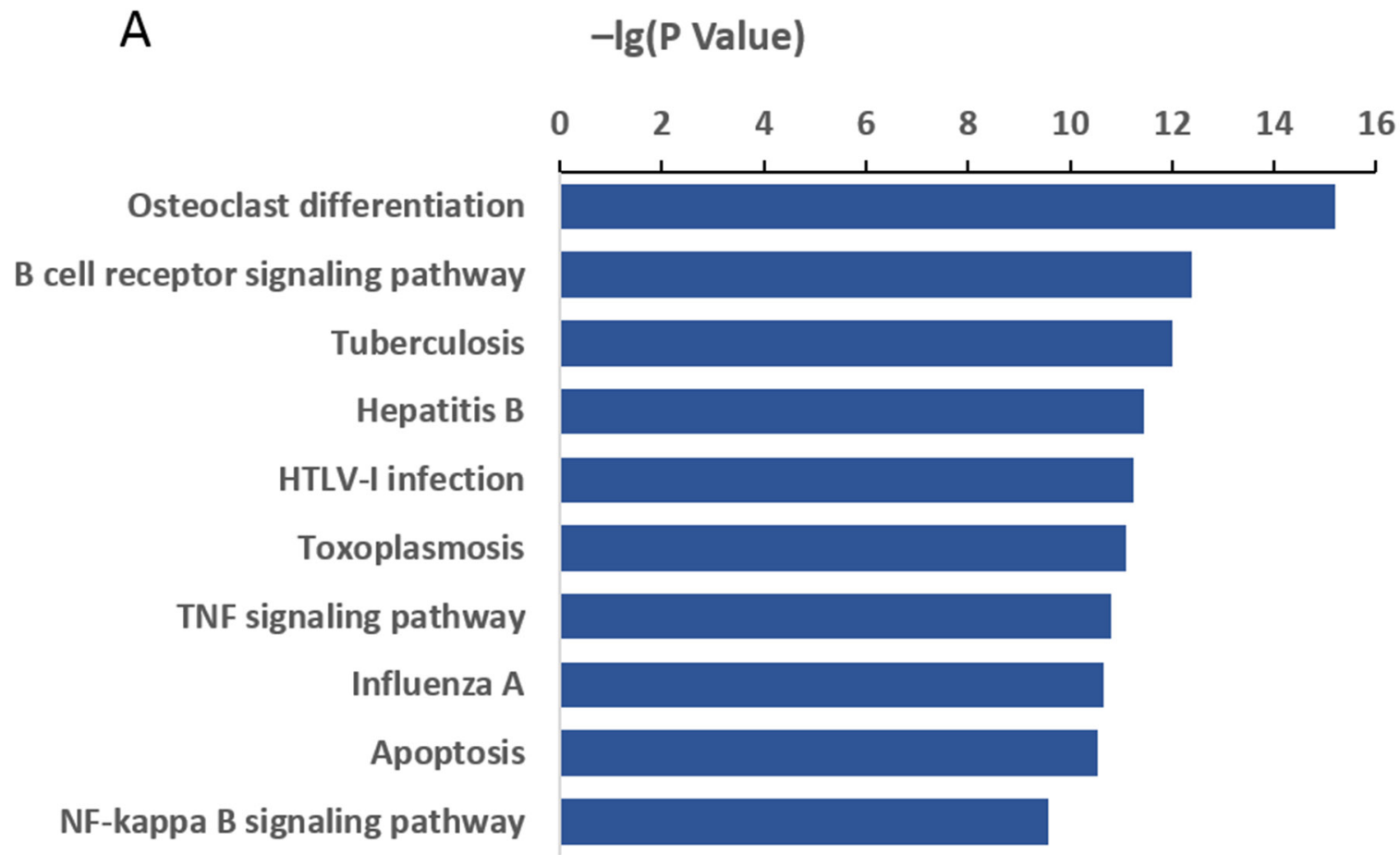

B $-\lg (P$ Value $)$

Metabolic pathways

Focal adhesion

Proteoglycans in cancer

Protein processing in endoplasmic...

Alzheimer's disease

Wnt signaling pathway

GnRH signaling pathway

Hippo signaling pathway

Non-alcoholic fatty liver disease (NAFLD)

Regulation of actin cytoskeleton
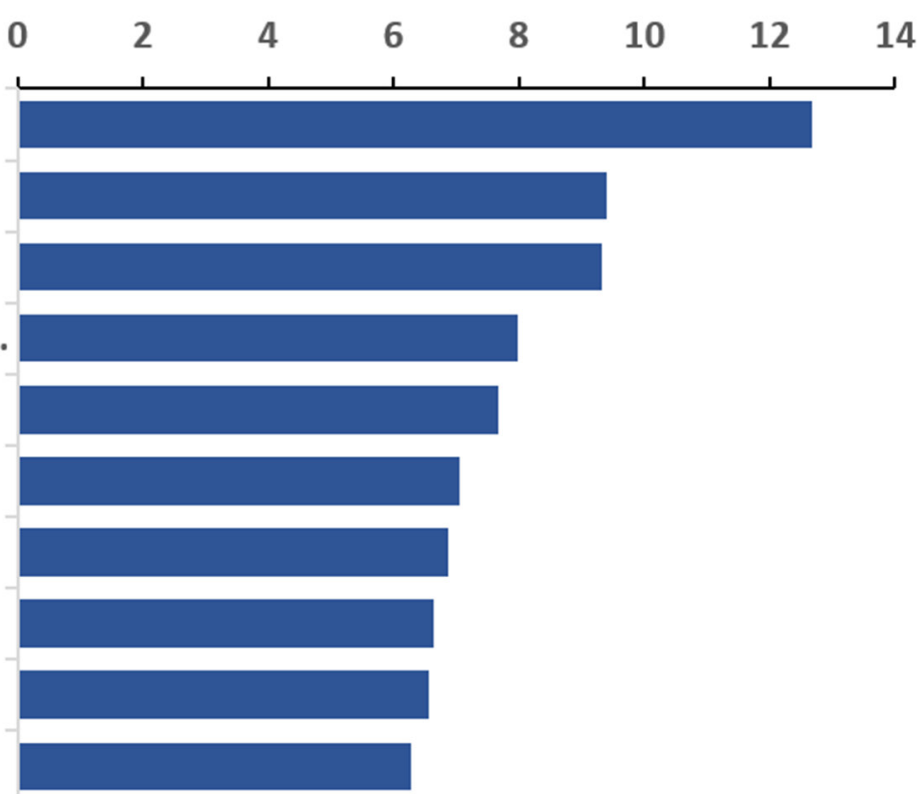

Figure 4: KEGG pathways of differentially expressed mRNAs. Vertical and horizontal axes represent the pathway category and enrichment score [-log 10 (P value)] of the pathway, respectively. (A) F0-HFD/F0-NC and (B) F1-HFD/F1-NC. 
with the G-protein coupled receptor signalling pathway, pancreatic polypeptide receptor activity, and lysine biosynthesis. The G-protein coupled receptor signalling pathway plays an important role in regulating mammalian reproduction and maintains oocyte meiosis by mitogenactivated protein kinase (MAPK) signalling [34]. Another study has demonstrated that a member of the pancreatic polypeptide family-Neuropeptide Y (NPY) could inhibit GnRH-1 neuronal activity through the $\mathrm{G}$ protein-coupled
Y1 receptor (Y1R) in the early developmental stage [35]. This suggests that intergenerational obesity is inherited by offspring through these pathways associated with growth and development.

LncRNAs modulate gene expression in cis or in trans $[36,37]$. In our study, two independent algorithms were used to predict the cis and trans target genes of differentially expressed lncRNAs in the sperm of obese mice. For example, the lncRNA showing the greatest

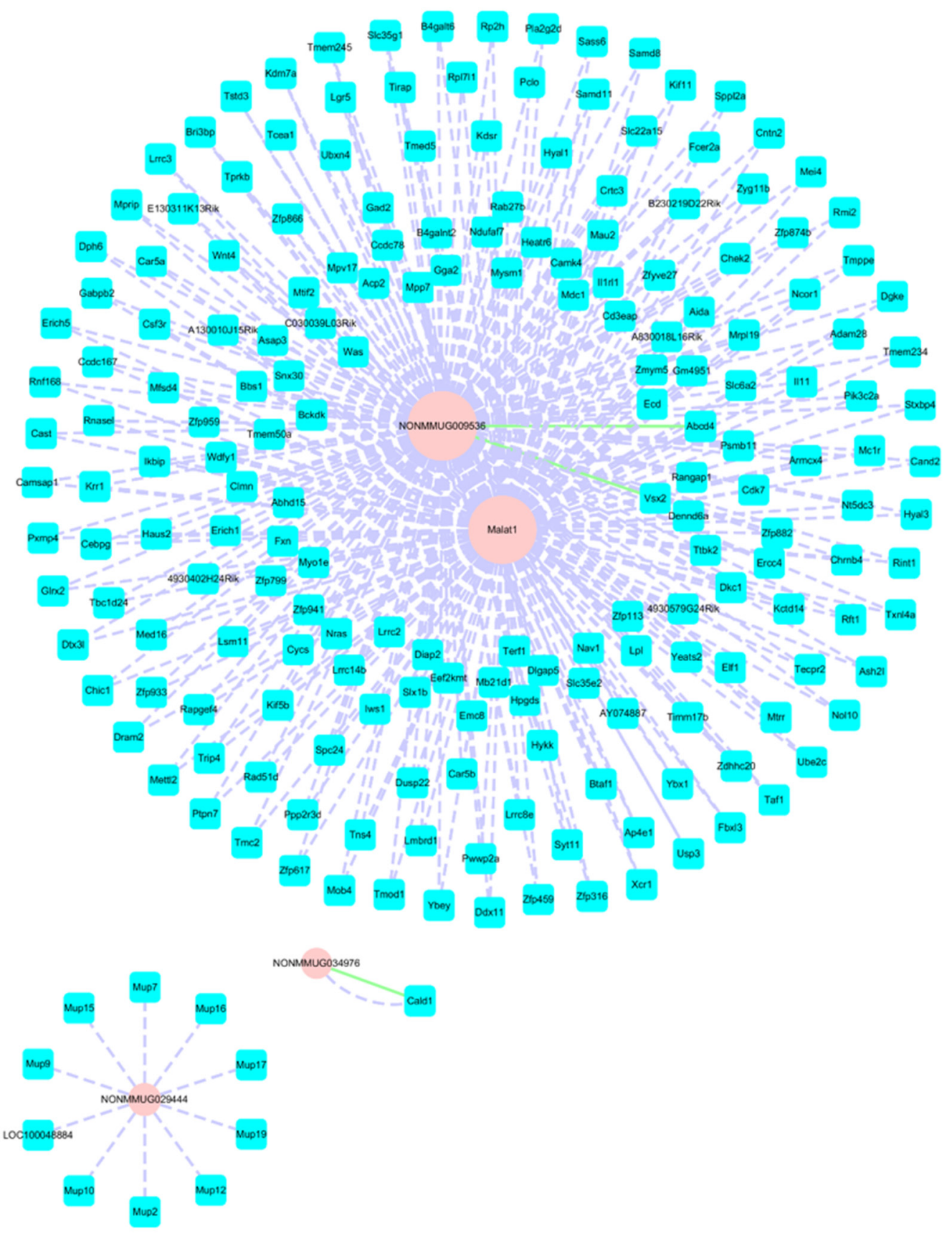

Figure 5: Coding-non-coding gene co-expression network (NONMMUG009526, Malat1, NONMMUG034976, and NONMMUG029444). Blue square and pink round nodes represent coding and non-coding genes; purple dashed and blue solid lines between the two nodes represent trans and cis targets, respectively. A larger point indicates that more targets are associated with the IncRNA. 

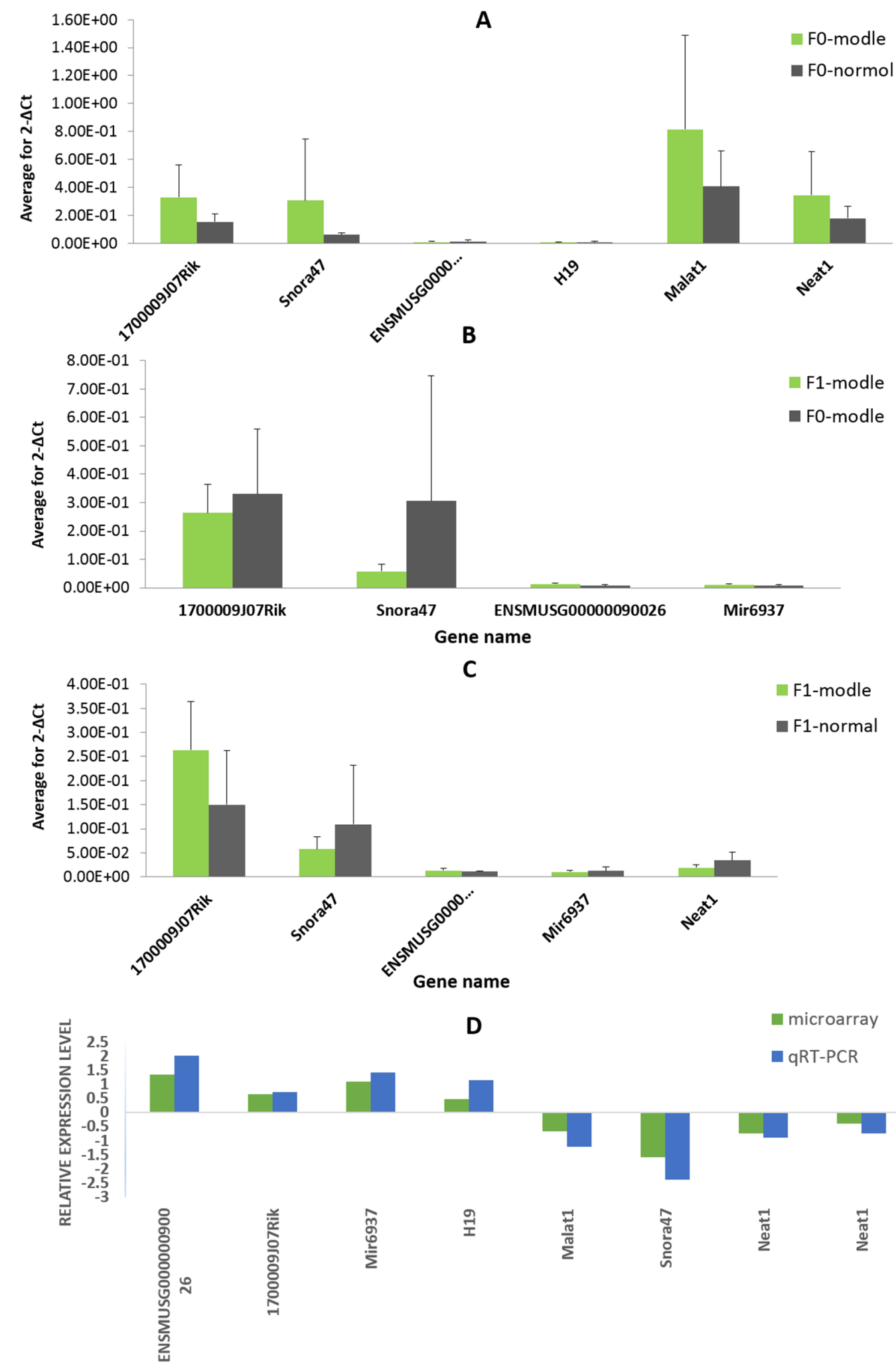

Figure 6: Validation of microarray data by quantitative reverse transcription-polymerase chain reaction (qRT-PCR). The relative expression levels of eight lncRNAs are shown comparing (A) F0-HFD/F0-normal; (B) F1-HFD/F0-HFD; and (C) F1-HFD/ F1-normal. Data are presented as average 2-ACt, $\mathrm{n}=10$. (D) Comparison between qRT-PCR results and microarray data revealing a good correlation of the two methods. The heights of the columns represent the fold changes ( $\log 2$ transformed) computed from the qPCR and microarray data. 


\begin{tabular}{|c|c|c|}
\hline Gene & & Primer sequence $\left(5^{\prime}\right.$ to $\left.3^{\prime}\right)$ \\
\hline \multicolumn{3}{|l|}{ Primers for lncRNAs } \\
\hline \multirow[t]{2}{*}{ H19 } & Forward & GTGTGGCCGTGTGCTTGAG \\
\hline & Reverse & GTAGGGCATGTTGAACACTTTATGA \\
\hline \multirow[t]{2}{*}{ 1700009J07Rik } & Forward & AGAAGCCAGCCACCACTAAG \\
\hline & Reverse & GACAGGACCAGCTTGCTTTC \\
\hline \multirow[t]{2}{*}{ Neat1 } & Forward & GGGAAAGCTGTTGGGTTGTA \\
\hline & Reverse & CGGCAGAATTTGTGGCTAAC \\
\hline \multirow[t]{2}{*}{ Snora47 } & Forward & CCGTGCTGCCTTCCATTG \\
\hline & Reverse & CCACGGTGATAGAGAGGACATTC \\
\hline \multirow[t]{2}{*}{ Malat1 } & Forward & TGCTGCATTAAGCCTGGAGT \\
\hline & Reverse & GAAACATTGGCACACTGCAC \\
\hline \multirow[t]{2}{*}{ Neat1 } & Forward & TGGGGAAATGTGAAGAAAGC \\
\hline & Reverse & TTGCTGTAAAGGGGAGGAAA \\
\hline \multirow[t]{2}{*}{ ENSMUSG00000090026 } & Forward & TTGACTACCTTTGGCACCG \\
\hline & Reverse & AAGCAAGCTCTGGTCTCTTCTG \\
\hline \multirow[t]{2}{*}{ Mir6937 } & Forward & GTAAGGGCTGGGTCTGTGTG \\
\hline & Reverse & CTAGTGCAAGGGGGAACCT \\
\hline \multicolumn{3}{|l|}{ Primers for internal control } \\
\hline \multirow[t]{2}{*}{ Gapdh(mouse) } & Forward & CACAATTTCCATCCCAGACC \\
\hline & Reverse & GTGGGTGCAGCGAACTTTAT \\
\hline
\end{tabular}

difference in expression between F0-HFD and F0-NC in the NONMMUG009536 microarray was predicted to act both in cis (ATP binding cassette subfamily $D$ member [Abcd] 4 and visual system homeobox 2) and in trans (Rad51d and Wnt4). Abcd4 is linked to vitamin B12 deficiency, which leads to a reduction in fat metabolism; the finding that $\mathrm{Abcd} 4$ was downregulated in the F0-HFD group $(\mathrm{F} 0-\mathrm{HFD} / \mathrm{F} 0-\mathrm{NC}=0.46)$ is consistent with the fact that it could cause or aggravate obesity [38]. Wnt4 may be involved in the pathogenesis of female infertility; spermatozoon-derived Wnt4 is transported to the fertilized egg and translated, and this process may be important in early embryonic development and provide a basis for the paternal gene effect [39]. Further studies are needed to confirm the functions of these differentially expressed lncRNAs and their regulation of cis and trans target genes in the sperm of obese mice offspring.

In conclusion, by analysing the expression of sperm lncRNA, we demonstrated that obesity was intergenerationally inherited by the offspring and similar expression patterns of lncRNAs in the F1 and F0 generation sperm may act as the hereditary vector mediating paternal heredity of obesity. Therefore, lncRNAs might serve as potential candidates for further comprehensive understanding and examination of obesityinduced male infertility as well as intergenerational inheritance of obesity.

\section{METHODS}

\section{Ethics statement}

The study protocol was approved by the Animal Care and Management Committee of the Beijing University of Chinese Medicine. All animal manipulations were according to the guidelines of the Animal Care Committee.

\section{Mice and diets}

Male C57bl/6J mice (8 weeks old; Hua Fu Kang Company, Beijing, China) were used in this study. After 1 week of adaptive feeding, the mice were randomly divided into obesity model (HFD) and non-obese (NC) groups (n $=10)$. HFD groups were continuously fed an HFD $(60 \%$ fat) for 10 weeks to induce obesity (body weight: 39-45 g; $\mathrm{n}=10$ ); after mating with fertile female mice, male offspring were reared for selection $(n=6)$. All mice other than half the F0 mice were maintained on SC feed. 


\section{Sperm collection}

Sperm was collected from F0-HFD $(n=6)$ and F0$\mathrm{NC}(\mathrm{n}=6)$ mice and their male offspring (F1-HFD $[\mathrm{n}=6]$ and F1-NC [ $\mathrm{n}=6]$, respectively). Mice were sacrificed by cervical dislocation, and sperm from the epididymis was transferred to a preheated human tubal fluid culture and centrifuged at 1,000 rpm for $5 \mathrm{~min}$. Sperm capacitation was measured for $30 \mathrm{~min}$, and the sperm supernatant was centrifuged again and collected.

\section{RNA extraction and purification}

Total RNA was extracted and purified using miRNeasy Mini kit (cat. no. 1038703; Qiagen, Hilden, Germany) following the manufacturer's instructions. RNA integration was analysed with a Bioanalyzer 2100 (Agilent Technologies, Santa Clara, CA, USA).

\section{RNA amplification and labelling}

Total RNA was amplified and labelled with the Low-Input Quick Amp WT Labeling kit (cat. no. 51902943; Agilent Technologies) following the manufacturer's instructions. Labelled cRNA was purified with the RNeasy Mini kit (cat. no. 74106).

\section{Microarray hybridisation}

Each slide was hybridised with $1.65 \mu \mathrm{g}$ Cy3-labelled cRNA using the Gene Expression Hybridization kit (cat. no. 5188-5242; Agilent Technologies) in a hybridisation oven (cat. no. G2545A; Agilent Technologies) according to the manufacturer's instructions. After $17 \mathrm{~h}$ of hybridisation, the slides were washed in staining dishes (cat. no. 121; Thermo Fisher Scientific, Waltham, MA, USA) using the Gene Expression Wash Buffer kit (cat. no. 5188-5327; Agilent Technologies) as instructed by the manufacturer.

\section{Data acquisition}

Slides were scanned with the Microarray Scanner (cat. no. G2565CA; Agilent Technologies) using the following default settings: dye channel $=$ green, scan resolution $=3$ $\mu \mathrm{m}, \mathrm{PMT}=100 \%$, and 20 bit. Data were extracted with Feature Extraction v.10.7 (Agilent Technologies). Raw data were normalised with the quantile algorithm of GeneSpring v.12.6.1 (Agilent Technologies). The microarray data presented in this article have been deposited in the National Center for Biotechnology Information Gene Expression Omnibus (GEO) and are accessible through GEO Series accession number GSE118039.

\section{Analysis of gene function}

The differentially expressed genes were input into the Database for Annotation, Visualization, and
Integrated Discovery (DAVID; http://david.abcc.ncifcrf. gov/) v.6.7, which used GO to identify the molecular functions represented in gene profiles and KEGG to analyse the potential functions of these genes in pathways $[40,41]$. Lower $P$ values represent more significant correlations; the recommended cut-off $P$ value was 0.05 .

\section{LncRNA target prediction}

Differentially expressed lncRNAs were selected for target prediction as previously described [42]. We used two independent algorithms to identify target genes. The first searched for those acting in cis. Using University of California Santa Cruz (UCSC) gene annotations (http://genome.ucsc.edu/), lncRNAs and potential target genes were paired and visualised using the UCSC genome browser. Those transcribed within a 10-kbp window up- or downstream of the IncRNA were considered as cis target genes. The second algorithm is based on mRNA sequence complementarity and RNA duplex energy prediction, and evaluated the impact of lncRNA binding on complete mRNA molecules using BLAST for first-round screening. RNAplex was used to screen target genes in trans [43] with the RNAplex parameter set as $-\mathrm{e}^{-20}$.

\section{Coding-non-coding gene co-expression network}

The majority of identified transcripts have unknown function. We constructed a codingnoncoding gene co-expression network that included the differentially expressed lncRNAs for cis and trans targeted coding genes from re-annotated Affymetrix Mouse Genome Array data.

\section{qRT-PCR}

Total RNA was extracted from additional sperm samples of F0-HFD, F0-NC, F1-HFD, and F1-SC mice $(\mathrm{n}=10$ each) using RNAiso Plus (Takara Bio, Dalian, China). The RNA was quantified using a NanoDrop 1000 spectrophotometer (Thermo Fisher Scientific, Waltham, MA, USA). The ratio of absorbances at 260 and $280 \mathrm{~nm}$ (A260/A280 $\geq 1.8$ ) was used to assess RNA purity. The RNA was then reverse transcribed using PrimeScript RT Master Mix (Takara Bio) according to the manufacturer's instructions. The eight differentially expressed lncRNAs were analysed by qRT-PCR using SYBR Premix Ex Taq II (Takara Bio) on the LightCycler 480 (Roche Diagnostics, Indianapolis, IN, USA) following the manufacturer's instructions, with glyceraldehyde 3-phosphate dehydrogenase (GAPDH) used as an internal control. Amplified genes and primers used in this study are shown in Table 3. The relative expression level of each lncRNA was calculated using double-standard curves. Experiments were performed in triplicate. 


\section{Statistical analysis}

Data are expressed as mean \pm standard deviation. The threshold value used to screen differentially expressed lncRNAs and mRNAs was fold change $>2.0(P<0.05)$. Differences between means were evaluated with the Student's $t$ test using SPSS v.22.0 (SPSS Inc., Chicago, IL, USA). $\mathrm{P}<0.05$ was considered statistically significant.

\section{Author contributions}

S.H. Gao and G.J. Jiang designed the experiments; T. An and T. Zhang wrote the manuscript;

F. Teng, J.C. Zuo, Y.Y. Pan, J.N. Miao, and N. Yu performed the experiments; Y.F. Liu, Y.J. Gu, D.D. Zhao, and F.F. Mo analysed the data. All authors reviewed the manuscript.

\section{ACKNOWLEDGMENTS}

This work was supported by Grants from the National Natural Science Foundation of China (NSFC 30770247), Beijing Municipal Education Commission scientific research and graduate training to build projects (1000062520025), Production and research joint cultivate project (1000062520181) and the International Cooperation Projects of MOE (2011DFA30920).

\section{CONFLICTS OF INTEREST}

The authors declare that they have no conflicts of interest in the study.

\section{REFERENCES}

1. Palmer NO, Bakos HW, Fullston T, Lane M. Impact of obesity on male fertility, sperm function and molecular composition. Spermatogenesis. 2012; 2:253.

2. Sharma R, Biedenharn KR, Fedor JM, Agarwal A. Lifestyle factors and reproductive health: taking control of your fertility. Reprod Biol Endocrinol. 2013; 11:66.

3. Rato L, Alves MG, Cavaco JE, Oliveira PF. Highenergy diets: a threat for male fertility? Obes Rev. 2014; 15:996-1007.

4. Du Plessis SS, Cabler S, Mcalister DA, Sabanegh E, Agarwal A. The effect of obesity on sperm disorders and male infertility. Nat Rev Urol. 2010; 7:153-61.

5. Agarwal A, Nandipati KC, Sharma RK, Zippe CD, Raina $\mathrm{R}$. Role of oxidative stress in the pathophysiological mechanism of erectile dysfunction. J Androl. 2006; 27:335-347.

6. Fullston T, Mcpherson NO, Owens JA, Kang WX, Sandeman LY, Lane M. Paternal obesity induces metabolic and sperm disturbances in male offspring that are exacerbated by their exposure to an "obesogenic" diet. Physiol Rep. 2015; 3:e12336.

7. Mcpherson NO, Fullston T, Aitken RJ, Lane M. Paternal obesity, interventions, and mechanistic pathways to impaired health in offspring. Ann Nutr Metab. 2014; 64:231

8. Ost A, Lempradl A, Casas E, Weigert M, Tiko T, Deniz M, Pantano L, Boenisch U, Itskov PM, Stoeckius M, Ruf M, Rajewsky N, Reuter G, et al. Paternal diet defines offspring chromatin state and intergenerational obesity. Cell. 2014; 159:1352-64.

9. Bohacek J, Mansuy IM. Molecular insights into transgenerational non-genetic inheritance of acquired behaviours. Nat Rev Genet. 2015; 16:641-652.

10. Siklenka K, Erkek S, Godmann M, Lambrot R, McGraw S, Lafleur C, Cohen T, Xia J, Suderman M, Hallett M, Trasler J, Peters AH, Kimmins S. Disruption of histone methylation in developing sperm impairs offspring health transgenerationally. Science. 2015; 350:aab2006.

11. Fullston T, Ohlsson Teague EM, Palmer NO, DeBlasio MJ, Mitchell M, Corbett M, Print CG, Owens JA, Lane M. Paternal obesity initiates metabolic disturbances in two generations of mice with incomplete penetrance to the F2 generation and alters the transcriptional profile of testis and sperm microRNA content. FASEB J. 2013; 27:4226.

12. de Carso Barbosa T, Ingerslev LR, Alm P, Versteyhe S, Massart J, Rasmussen M, Donkin I, Sjögren R, Mudry JM, Vetterli L, Gupta S, Krook A, Zierath JR, Barrès R. Highfat diet reprograms the epigenome of rat spermatozoa and transgenerationally affects metabolism of the offspring. Mol Metab. 2016; 5:184-197.

13. Moore GE, Stanier P. Fat dads must not be blamed for their children's health problems. BMC Med. 2013; 11:30.

14. Youngson NA, Lecomte V, Maloney CA, Leung P, Liu J, Hesson LB, Luciani F, Krause L, Morris MJ. Obesityinduced sperm DNA methylation changes at satellite repeats are reprogrammed in rat offspring. Asian J Androl. 2015; 18:930.

15. Gapp K, Jawaid A, Sarkies P, Bohacek J, Pelczar P, Prados J, Farinelli L, Miska E, Mansuy IM. Implication of sperm RNAs in transgenerational inheritance of the effects of early trauma in mice. Nat Neurosci. 2014; 17:667-669.

16. Monnier P, Martinet C, Pontis J, Stancheva I, Ait-Si-Ali S, Dandolo L. H19 IncRNA controls gene expression of the Imprinted Gene Network by recruiting MBD1. Proc Natl Acad Sci U S A. 2013; 110:20693.

17. Chen J, Cui X, Shi C, Chen L, Yang L, Pang L, Zhang J, Guo X, Wang J, Ji C. Differential IncRNA expression profiles in brown and white adipose tissues. Mol Genet Genomics. 2015; 290:699-707.

18. Chen J, Liu Y, Lu S, Yin L, Zong C, Cui S, Qin D, Yang Y, Guan Q, Li X, Wang X. The role and possible mechanism of IncRNA U90926 in modulating 3T3-L1 preadipocyte differentiation. Int J Obes (Lond). 2016; 41:299-308. 
19. Zhang C, Gao L, Xu EY. LncRNA, a new component of expanding RNA-protein regulatory network important for animal sperm development. Semin Cell Dev Biol. 2016; 59:110-117.

20. Luk CS, Gao H, Xiao S, Liao J, Wang D, Tu J, Rennert OM, Chan WY, Lee TL. GermlncRNA: a unique catalogue of long non-coding RNAs and associated regulations in male germ cell development. Database (Oxford). 2014; 2015:bav044.

21. Jiang GJ, Teng Z, Tian A, Zhao DD, Yang XY, Zhang DW, Zhang Y, Mu QQ, Yu N, Ma XS, Gao SH. Differential expression of long noncoding RNAs between sperm samples from diabetic and non-diabetic mice. Plos One. 2016; 11:e0154028.

22. Gao F, Zhang P, Zhang H, Zhang Y, Zhang Y, Hao Q, Zhang $X$. Dysregulation of long noncoding RNAs in mouse testes and spermatozoa after exposure to cadmium. Biochem Biophys Res Commun. 2017; 484:8-14.

23. Jia S, Ruan Y, Ming W, Chen R, Yu N, Sun L, Liu T, Chen H. Differentially expressed circulating LncRNAs and mRNA identified by microarray analysis in obese patients. Sci Rep. 2016; 6:35421.

24. Schlitt T, Palin K, Rung J, Dietmann S, Lappe M, Ukkonen E, Brazma A. From gene networks to gene function. Genome Res. 2003;13:2568-76.

25. Grandjean V, Fourré S, Abreu DA, Derieppe MA, Remy JJ, Rassoulzadegan M. RNA-mediated paternal heredity of diet-induced obesity and metabolic disorders. Sci Rep. 2015; 5:18193.

26. Fox H, Lamond AI. Paraspeckles. Cold Spring Har Perspect Biol. 2010; 2:a000687.

27. Nakagawa S, Ip JY, Shioi G, Tripathi V, Zong X, Hirose T, Prasanth KV. Malat1 is not an essential component of nuclear speckles in mice. RNA. 2012; 18:1487-1499.

28. Cohen A, Dempster DW, Recker RR, Lappe JM, Zhou H, Zwahlen A, Müller R, Zhao B, Guo X, Lang T, Saeed I, Liu XS, Guo XE, et al. Abdominal fat is associated with lower bone formation and inferior bone quality in healthy premenopausal women: a transiliac bone biopsy study. J Clin Endocrinol Metab. 2013; 98:2562-2572.

29. Bredella MA, Fazeli PK, Lecka-Czernik B, Rosen CJ, Klibanski A. IGFBP-2 is a negative predictor of coldinduced brown fat and bone mineral density in young nonobese women. Bone. 2013; 53:336-339.

30. Li L, Wojtowicz JL, Malin JH, Huang T, Lee EB, Chen Z. GnRH-mediated olfactory and visual inputs promote mating-like behaviors in male zebrafish. PloS One. 2017; 12:e0174143.
31. Vastagh C, Rodolosse A, Solymosi N, Liposits Z. Altered expression of genes encoding neurotransmitter receptors in GnRH neurons of proestrous mice. Front Cell Neurosci. 2016; 10:230.

32. Evans MC, Anderson G. Neuroendocrine integration of nutritional signals on reproduction. J Mol Endocrinol. 2017; 58:R107-R128.

33. Ye H, Li X, Zheng T, Hu C, Pan Z, Huang J, Li J, Li W, Zheng Y. The hippo signaling pathway regulates ovarian function via the proliferation of ovarian germline stem cells. Cell Physiol Biochem. 2017; 41:1051-1062.

34. Thomas P. Role of G-protein-coupled estrogen receptor (GPER/GPR30) in maintenance of meiotic arrest in fish oocytes. J Steroid Biochem Mol Biol. 2016; 167:153-161.

35. Klenke U, Constantin SS, Wray S. Neuropeptide Y directly inhibits neuronal activity in a subpopulation of gonadotropin-releasing hormone-1 neurons via Y1 receptors. Endocrinology. 2010; 151:2736.

36. Roberts TC, Morris KV, Weinberg MS. Perspectives on the mechanism of transcriptional regulation by long non-coding RNAs. Epigenetics. 2013; 9:13.

37. Wang L, Fu D, Qiu Y, Xing X, Xu F, Han C, Xu X, Wei Z, Zhang Z, Ge J, Cheng W, Xie HL. Genome-wide screening and identification of long noncoding RNAs and their interaction with protein coding RNAs in bladder urothelial cell carcinoma. Cancer Lett. 2014; 349:77-86.

38. Coelho D, Kim JC, Miousse IR, Fung S, du Moulin M, Buers I, Suormala T, Burda P, Frapolli M, Stucki M, Nürnberg $\mathrm{P}$, Thiele $\mathrm{H}$, Robenek $\mathrm{H}$, et al. Mutations in ABCD4 cause a new inborn error of vitamin B12 metabolism. Nat Genet. 2012; 44:1152-1155.

39. Mafra F, Catto M, Bianco B, Barbosa CP, Christofolini D. Association of WNT4 polymorphisms with endometriosis in infertile patients. J Assist Reprod Genet. 2015; 32:1359-1364.

40. Huang da W, Sherman BT, Lempicki RA. Bioinformatics enrichment tools: paths toward the comprehensive functional analysis of large gene lists. Nucleic Acids Res. 2009; 37:1-13.

41. Huang da W, Sherman BT, Lempicki RA. Systematic and integrative analysis of large gene lists using DAVID bioinformatics resources. Nat Protoc. 2009; 4:44-57.

42. Han L, Zhang K, Shi Z, Zhang J, Zhu J, Zhu S, Zhang A, Jia Z, Wang G, Yu S, Pu P, Dong L, Kang C. LncRNA profile of glioblastoma reveals the potential role of lncRNAs, in contributing to glioblastoma pathogenesis. Int J Oncol. 2012; 40:2004.

43. Tafer H, Hofacker IL. RNAplex: a fast tool for RNA-RNA interaction search. Bioinformatics. 2008; 24:2657-2663. 